\title{
A Lower Bound for Odd Triperfects
}

\section{By Walter E. Beck and Rudolph M. Najar}

Abstract. If $\mathbf{n}$ is odd and $\sigma(n)=3 n$, then $\mathbf{n}$ exceeds $10^{50}$.

A natural number $\mathbf{n}$ is called triperfect if $\sigma(n)=3 n$. Six triperfects are known, all even. The existence of an odd triperfect is an open question. Kanold [1] showed that an odd triperfect: (a) has at least nine distinct prime factors; (b) is a square; and, (c) exceeds $10^{20}$. The purpose of this note is to improve the last result. We prove

THEOREM. If $\mathbf{n}$ is odd triperfect, then $\mathbf{n}$ exceeds $10^{50}$.

The inequality $\sigma\left(p^{a}\right) / p^{a}<p /(p-1)$, where $x /(x-1)$ is monotone decreasing in $\mathbf{x}$, yields the least number of primes so that $\sigma(n) / n \geqslant 3$. Thus,

LEMMA. If $n$ is triperfect and $(n, 6)=1$, then $\mathbf{n}$ exceeds $10^{108}$ and has at least 32 distinct prime factors.

Assume $\mathrm{n}$ an odd triperfect and $3 \mid n$. Since $\sigma(n)=3 n$, even powers of prime factors of $\sigma(n)$ divide $\mathbf{n}$, up to, possibly, powers of 3 . This caution is necessary since if $3^{a} \| n$, then $3^{a+1} \| \sigma(n)$. If $p^{a} \| n$, then $p^{a} \sigma\left(p^{a}\right) \mid n$, again, up to power of 3 . As $3^{54}>5 \cdot 10^{25}$, it suffices to examine $n$ assuming $3^{t} \| n, t$ even, $2 \leqslant t<54$.

We construct all sequences of primes $P=\left\{3=p_{0}, p_{1}, p_{2}, \ldots\right\}$ and of even naturals $N=\left\{t=n_{0}, n_{1}, n_{2}, \ldots\right\}$ so that (a) $p_{i+1}$ is the largest prime divisor of $\sigma\left(p_{i}^{n_{i}}\right)$, and (b) for each $i, n_{i}=2,4,6, \ldots$ The sequences are extended until either: (a) the product of known factors of $n$ exceeds $10^{50}$, (b) powers of 3 among the $\sigma$ 's exceed $t+1$, or (c) $\mathbf{n}$ is shown to be triperfect. For the most part, complete prime factorizations of the $\sigma$ 's were calculated, although this was not always necessary. Of the 221 cases that occurred, four were of type (b) and the remainder of type (a). No triperfects were found. This proves the theorem.

From known results on perfect and multiperfect numbers, we have

COROllaRY. If $\mathbf{n}$ is odd multiperfect, the $\mathbf{n}$ exceeds $10^{50}$.

Factorizations were computer generated. The authors acknowledge with gratitude the assistance of Dr. Jacob Gerlach, Department of Mathematics, University of Wisconsin-Whitewater, and of Professor D. H. Lehmer, Department of Mathematics, University of California-Berkeley. The authors also wish to acknowledge the valuable suggestions of the referee.

Received January 12, 1981; revised June 22, 1981.

1980 Mathematics Subject Classification. Primary 10A20.

Key words and phrases. Triperfects, odd triperfects. 
A copy of the computational data will be deposited with UMT. The Appendix below contains the computations for the cases $3^{16}\left\|n, 3^{24}\right\| n$, and $3^{44} \| n$.

\section{Appendix.}

Case 16-1

$$
\begin{gathered}
3^{16}=43046721 ; \sigma\left(3^{16}\right)=64570081=1871 \cdot 34511 \\
34511^{2}=1191009121 ; \sigma\left(34511^{2}\right)=1191043033=13 \cdot 19 \cdot 4822039 . \\
4822039^{2}=23252060117521 ; \\
\sigma\left(4822039^{2}\right)=23252064939561=3 \cdot 331 \cdot 23415976777 .
\end{gathered}
$$

Known factors include $\left[\sigma\left(3^{16}\right)\right]^{2}>3 \cdot 10^{15} ; \quad\left[\sigma\left(34511^{2}\right)\right]^{2}>10^{18}$;

$$
\left[\sigma\left(4822039^{2}\right)\right]^{2}>4 \cdot 10^{26} \text {. Exceed } 10^{59} \text {. }
$$

Case 16-2

$$
4822039^{4}>10^{26} . \quad \sigma\left(4822039^{4}\right)>10^{26} . \text { Exceed } 10^{52} .
$$

Case 16-3

$$
34511^{4}=1418502726305192641 ;
$$

$\sigma\left(34511^{4}\right)=1418543830412011105=5 \cdot 11 \cdot 31 \cdot 71 \cdot 131 \cdot 89451727381$.

Known factors include $34511^{4}>10^{18} ; \quad\left[\sigma\left(34511^{4}\right)\right]^{2}>10^{36}$.

Exceed $10^{54}$.

Case 16-4

$$
34511^{6}>10^{27} ; \quad \sigma\left(34511^{6}\right)>10^{27} . \text { Exceed } 10^{54} \text {. }
$$

\section{End $3^{16}$.}

Case 24-1

$$
\begin{aligned}
& 3^{24}=282429536481 ; \quad \sigma\left(3^{24}\right)=423644304721=11^{2} \cdot 8951 \cdot 391151 \\
& 391151^{2}=152999104801 ; \quad \sigma\left(391151^{2}\right)=152999495953 \\
& =313 \cdot 488816281
\end{aligned}
$$

Known factors include $3^{24}>10^{11} ;\left[\frac{1}{11} \sigma\left(3^{24}\right)\right]^{2}>10^{20}$;

$$
\left[\sigma\left(391151^{2}\right)\right]^{2}>10^{22} \text {. Exceed } 10^{53} \text {. }
$$

Case 24-2

$$
\begin{aligned}
391151^{4} & =23408726069907381249601 ; \\
\sigma\left(391151^{4}\right) & =23408785915813222761505 \\
& =5 \cdot 4681757183162644552301 .
\end{aligned}
$$

The primality of the underscored integer is unknown. $3 \nmid \sigma\left(391151^{4}\right)$.

Known factors include $3^{24}>10^{11} ; 391151^{4}>10^{22}$;

$$
\sigma\left(391151^{4}\right)>10^{22} \text {. Exceed } 10^{55} \text {. }
$$


Case 24-3

$$
\begin{gathered}
391151^{6}>10^{30} ; \quad \sigma\left(391151^{6}\right)>10^{30} . \\
\text { End } 3^{24} .
\end{gathered}
$$

Case 44-1

$$
\begin{gathered}
3^{44}=984770902183611232881 ; \\
\sigma\left(3^{44}\right)=1477156353275416849321 \\
=11^{2} \cdot 13 \cdot 181 \cdot 757 \cdot 1621 \cdot 4561 \cdot 927001 . \\
\text { Known factors include } 3^{44}>10^{20} ;\left[\frac{1}{11}\left(3^{44}\right)\right]^{2}>10^{40} . \text { Exceed } 10^{60} .
\end{gathered}
$$

End $3^{44}$.

Department of Mathematics

University of Northern Iowa

Cedar Falls, Iowa 50613

Department of Mathematics

University of Wisconsin-Whitewater

Whitewater, Wisconsin 53190

1. H.-J. KANOLD, “Über mehrfach volkommene Zahlen. II," J. Reine Angew. Math., v. 197, 1957, pp. 82-96. MR 18, 873. 